\title{
Effect of bamboo grass (Tiliacora triandra, Diels) pellet supplementation on rumen fermentation characteristics and methane production in Thai native beef cattle
}

\author{
Chinda Wann', Metha Wanapat ${ }^{1, *}$, Chaowarit Mapato ${ }^{1}$, Thiwakorn Ampapon', and Bi-zhi Huang ${ }^{2}$
}

\section{* Corresponding Author: Metha Wanapat Tel: +66-83-1-932-4180, Fax: +66-43-202368 E-mail: metha@kku.ac.th}

'Tropical Feed Resources Research and Development Center (TROFREC), Department of Animal Science, Faculty of Agriculture, Khon Kaen University, Khon Kaen 40002, Thailand

${ }^{2}$ Yunnan Academy of Grassland and Animal Science, Kunming 650212, Yunnan, China

\section{ORCID}

Chinda Wann

https://orcid.org/0000-0003-2846-8804 Metha Wanapat

https://orcid.org/0000-0002-7633-052X

Chaowarit Mapato

https://orcid.org/0000-0002-9368-3298

Thiwakorn Ampapon

https://orcid.org/0000-0002-5540-0939

Bi-zhi Huang

https://orcid.org/0000-0003-4308-2838

Submitted Sept 13, 2018; Revised Nov 14, 2018; Accepted Dec 15, 2018
Objective: The objective of this study was to investigate the effect of bamboo grass (Tiliacora triandra, Diels) pellet (Bamboo-Cass) supplementation on feed intake, nutrient digestibility, rumen microbial population and methane production in Thai native beef cattle.

Methods: Four Thai native beef cattle bulls ( $190 \pm 2 \mathrm{~kg}$ ) were randomly allotted to four respective dietary treatments in a $4 \times 4$ Latin square design. Treatments were the varying levels of Bamboo-Cass supplementation at 0, 50,100, and $150 \mathrm{~g} / \mathrm{head} / \mathrm{d}$, respectively. Rice straw was fed ad libitum and the concentrate offered at $0.5 \%$ of body weight.

Results: Under this experiment, the findings revealed that acetate and butyrate production were decreased $(\mathrm{p}<0.05)$, propionate increased $(\mathrm{p}<0.05)$, whilst ruminal $\mathrm{NH}_{3}-\mathrm{N}$ concentration was decreased $(\mathrm{p}<0.05)$ by supplementation of Bamboo-Cass at $150 \mathrm{~g} / \mathrm{head} / \mathrm{d}$. Moreover, rice straw intake, and microbial population were linearly increased $(\mathrm{p}<0.05)$, while methane production was decreased $(\mathrm{p}<0.05)$.

Conclusion: The results from the present study indicate that supplementation of BambooCass at $150 \mathrm{~g} / \mathrm{head} / \mathrm{d}$ significantly enhanced feed intake, decreased protozoa and increased bacterial population, rumen fermentation efficiency while decreased methane production. Therefore, Bamboo-Cass as a supplement is promising as a rumen enhancer in beef cattle fed on rice straw.

Keywords: Rumen Fermentation; Bamboo-Cass; Rice Straw; Methane Production

\section{INTRODUCTION}

Greenhouse gases including $\mathrm{CH}_{4}, \mathrm{CO}_{2}$, and $\mathrm{N}_{2} \mathrm{O}$ are emitted from rumen fermentation and results in a loss of dietary energy intake accounting up to $12 \%$. There are many possible $\mathrm{CH}_{4}$ mitigation strategies but one of the most promising implementations could be the dietary manipulating option which has been reported in the previous studies [1,2]. There have been numerous reports on the potential use of plant secondary compounds, particularly the use of condensed tannins and crude saponins on rumen microorganisms and the consequent fermentation end-products in mitigating rumen $\mathrm{CH}_{4}$ production $[3,4]$.

Bamboo grass (Tiliacora triandra, Diels) is a tropical climbing plant with abundant green leaves and yellow flower, belonging to Menispermaceae family. It has been known as a local resource plant in sub-tropical and tropical regions, used for medicine, cosmetics, and household products $[5,6]$. Bamboo grass is rich in phenolic compounds which are useful as a source of bioactive compounds [7]. Previous studies were conducted using this plant to investigate their antioxidant activities, and bioactive role as a medical plant $[6,8,9]$. Bamboo grass contains plant secondary compounds of condensed tannins ( $2.3 \%$ of dry matter [DM]) and crude saponins ( $1.3 \%$ of DM), and is potential feed resource for use as a strategic supple- 
ment in ruminant production [10,11]. However, information on the potential use of this plant in ruminant feeding is not yet available. Therefore, the objective of this study was to determine the effect of bamboo grass pellet (Bamboo-Cass) on feed intake, nutrient digestibility, rumen microbial population, and methane production in Thai beef cattle fed on rice straw.

\section{MATERIALS AND METHODS}

\section{The experimental area and animals care}

This experiment was done at the Ruminant Nutrition and Metabolism Research Center, Tropical Feed Resource Research and Development Center (TROFREC), Department of Animal Science, Faculty of Agriculture, Khon Kaen University (KKU), Khon Kaen, Thailand. All applicable international, national, and/or institutional guidelines for the care and use of animals were followed and allowed by the Khon Kaen University Animal Ethics Committee, ancillary to the Ethic of animal Experimentation of National Research Council of Thailand.

\section{Animals, feeds and experimental design}

All experimental beef cattle were treated with vitamin $\mathrm{AD}_{3} \mathrm{E}$ injection $(20 \mathrm{~mL} / \mathrm{head})$ and dewormed before imposing the respective treatments. They were raised in individual pens of $3 \times 4 \mathrm{~m}$ size where the water and mineral block were always available. Four Thai native beef cattle bulls $(190 \pm 2 \mathrm{~kg})$ were randomly assigned to receive four dietary treatments in a $4 \times 4$ Latin square design and were raised in animal pens with permanent roof. Animals were fed ad libitum with rice straw and water supply. The $14 \%$ crude protein $(\mathrm{CP})$ of concentrate was formulated and provided to all animals at $0.5 \%$ body weight (BW). All animals received four varying levels of Bamboo-Cass supplementation at $0,50,100$, and $150 \mathrm{~g} / \mathrm{head} / \mathrm{d}$, respectively. Each of the periods lasted for 21 days, the first 14 days was for adaptation and measurement of intake, while the last 7 days was for sample collections when the experimental animals were moved to the metabolism crates for total collections.

Bamboo grass (Tiliacora triandra, Diels) was harvested from fresh leaves from the climbing tree and sun-dried for use in the experiment. Bamboo grass pellets were made by combining bamboo grass (90\%), cassava chip (9\%), and molasses (1\%) (Bamboo-Cass) then sun-dried to achieve at least 85\% DM before storage for later feeding to the experimental animals [12].

\section{Data collection and sampling procedures}

All procedures and collection details of the feeds, urine, rumen fluid, blood samples, and rumen methane calculation were done as described by [13]. Animals were fed twice daily at 08:00 hours in the morning and 16:00 hours in the after- noon. Feed offered and refusal feeds were recorded throughout the experimental period for calculating feed intake. Feed and fecal samples were collected during the 7 days feeding period when the animals were in the metabolism crates for analyzing DM, for each period then stored at $-20^{\circ} \mathrm{C}$ for later chemical analysis. The samples were divided into two parts, the first part was analyzed for DM, and the second part kept for analysis of ash, CP, and acid detergent fiber (ADF) [14], neutral detergent fiber (NDF) [15]. The content of condensed tannins of bamboo grass and Bamboo-Cass were analyzed by using the modified vanillin-HCL method [16]. Crude saponins were analyzed by using methanol extraction [17].

Rumen fluid was taken from the middle part of rumen at 0 and 4 hour post morning feeding on the last day of each period prior to morning feeding using a tube connected with the vacuum pump. Rumen fluid was measured immediately for $\mathrm{pH}$ (HANNA Instruments HI 8424 microcomputer, Singapore) and strained through four layers cheesecloth. Fluid sample was divided into three parts for continuing analysis. The first $45 \mathrm{~mL}$ of rumen fluid sample was collected and kept in plastic bottle to which $5 \mathrm{~mL}$ of $1 \mathrm{M} \mathrm{H}_{2} \mathrm{SO}_{4}$ were added for stopping the process of microbial fermentation and then centrifuged with $3,000 \times \mathrm{g}$ for 10 minutes for volatile fatty acids (VFAs) analysis in the laboratory using high-performance liquid chromatography instruments by water and Novapak model 600E; water mode 1484UV detector; column Novapak C18; column size $3.9 \times 300 \mathrm{~mm}$; mobile phase $10 \mathrm{mM} \mathrm{H} 2 \mathrm{PO} 4$ ( $\mathrm{pH}$ 2.5) [18] and $\mathrm{NH}_{3}-\mathrm{N}$ analysis [14]. The second part (1 $\mathrm{mL}$ of rumen fluid) was quickly fixed with $10 \%$ of formalin solution ( $1: 9 \mathrm{v} / \mathrm{v}$, rumen fluid:10\% formalin) in plastic bottles for measurement of the microbial population. The total direction counts, method of microbial populations were calculated based on the use of a haemacytometer (Boeco, Singapore) [19]. The third part (10 mL of rumen fluid) was used for variable bacteria counts (cellulolytic, proteolytic, and amylolytic) using the roll-tube technique [20].

Blood samples were collected (about $10 \mathrm{~mL}$ ) from the jugular vein of all experimental animals and then transferred to tubes containing ethylenediaminetetraacetic acid at the same time as the collection of rumen fluid at 0 and 4 hour post morning feeding. Blood samples were quickly put in the refrigerator for 1 hour and then centrifuged for $20 \mathrm{~min}$ to separate plasma from the whole blood. Plasma was stored at $-20^{\circ} \mathrm{C}$ for later analysis of blood urea nitrogen (BUN) [21].

Calculation of ruminal $\mathrm{CH}_{4}$ production was estimated by using VFA proportions by $\mathrm{CH}_{4}$ production $=0.45\left(\right.$ acetate, $\left.\mathrm{C}_{2}\right)$ -0.275 (propionate, $\mathrm{C}_{3}$ ) +0.4 (butyrate, $\mathrm{C}_{4}$ ) [22] .

\section{Statistical analysis}

All data were statistically analyzed in a $4 \times 4$ Latin square design using general linear model procedure [23] according to the model: $\mathrm{Y}_{\mathrm{ijk}}=\mu+\mathrm{M}_{\mathrm{i}}+\mathrm{A}_{\mathrm{j}}+\mathrm{P}_{\mathrm{k}}+\varepsilon_{\mathrm{ijk}} ;$ where $\mathrm{Y}_{\mathrm{ijk}}=$ observation 
from animal $j$, receiving diet $i$, in the period $k ; \mu=$ the overall mean; $M_{i}=$ effect of treatment; $A_{j}=$ effect of animal; $P_{k}=$ effect of period, and $\varepsilon_{\mathrm{ijk}}=$ residual effect. Mean separations with a significantly different value $(\mathrm{p}<0.05)$ and difference between treatment means determined by Duncan's new multiple range test [24]. Trend of Bamboo-Cass level responses was performed by using the orthogonal polynomial.

\section{RESULTS}

\section{Chemical composition in feed ingredients}

Table 1 presents the data of concentrate ingredients and chemical composition of the experimental feeds. The concentrate was formulated using high level of cassava chip (61\%) as the main energy source, oil palm meal (20\%) and urea (2.5\%) as protein and non-protein nitrogen source, respectively. Important concentrations of nutrients such as $\mathrm{CP}$, NDF for concentrate, rice straw, Bamboo grass and Bamboo-Cass were $14.3 \%, 27.6 \%$; $2.1 \%, 75.1 \%$; $16.0 \%, 61.9 \%$; $14.7 \%$, 56.6\%, re-

Table 1. Feed ingredients of concentrate and chemical composition of the feeds

\begin{tabular}{|c|c|c|c|c|}
\hline Items & Concentrate & $\begin{array}{l}\text { Rice } \\
\text { straw }\end{array}$ & $\begin{array}{c}\text { Bamboo } \\
\text { grass }\end{array}$ & $\begin{array}{c}\text { Bamboo- } \\
\text { Cass }\end{array}$ \\
\hline \multicolumn{5}{|c|}{ Feed ingredients, $\%$ as fresh basis } \\
\hline Cassava chip & 61.0 & - & - & - \\
\hline Coconut meal & 12.0 & - & - & - \\
\hline Rice bran & 1.0 & - & - & - \\
\hline Palm meal & 20.0 & - & - & - \\
\hline Urea & 2.5 & - & - & - \\
\hline Molasses & 2.0 & - & - & - \\
\hline Sulphur & 0.5 & - & - & - \\
\hline Premix ${ }^{1)}$ & 0.5 & - & - & - \\
\hline Salt & 0.5 & - & - & - \\
\hline Total & 100 & - & - & - \\
\hline \multicolumn{5}{|l|}{ Chemical compositions } \\
\hline \multirow[t]{2}{*}{ DM (\%) } & 87.5 & 88.5 & 35.7 & 87.0 \\
\hline & & $--\%$ of $d r$ & matter----. & --------- \\
\hline OM & 94.2 & 87.9 & 92.5 & 94.7 \\
\hline$C P$ & 14.3 & 2.1 & 16.0 & 14.7 \\
\hline NDF & 27.6 & 75.1 & 61.9 & 56.6 \\
\hline ADF & 18.2 & 55.6 & 40.3 & 36.7 \\
\hline Condensed tannins & - & - & 3.1 & 2.8 \\
\hline Crude saponins & - & - & 1.4 & 1.3 \\
\hline \multicolumn{5}{|l|}{ Minerals (\%) } \\
\hline Calcium & - & - & - & 1.5 \\
\hline Phosphorus & - & - & - & 0.1 \\
\hline Potassium & - & - & - & 2.6 \\
\hline Sodium & - & - & - & 0.1 \\
\hline Magnesium & - & - & - & 0.4 \\
\hline
\end{tabular}

Bamboo-Cass, Bamboo grass pellet; DM, dry matter; OM, organic matter; $C P$, crude protein; NDF, neutral detergent fiber; $A D F$, acid detergent fiber.

1) Contains per kilogram: 4,000,000 IU vitamin $\mathrm{A} ; 400,000 \mathrm{IU}$ vitamin $\mathrm{D}_{3} ; 4,000 \mathrm{IU}$ vitamin $\mathrm{E} ; 0.002 \mathrm{~g}$ vitamin $\mathrm{B}_{12} ; 16 \mathrm{~g} \mathrm{Mn} ; 24 \mathrm{~g} \mathrm{Fe} ; 10 \mathrm{~g} \mathrm{Zn;} 2 \mathrm{~g} \mathrm{Cu} ; 0.05 \mathrm{~g} \mathrm{Se} ; 0.2$ $\mathrm{g} C 0,0.5 \mathrm{~g} \mathrm{l}$. spectively. Plant secondary compounds namely condensed tannins and crude saponins for bamboo grass and BambooCass were $3.1 \%, 1.4 \% ; 2.8 \%, 1.3 \%$, respectively.

\section{Effect of Bamboo-Cass supplementation on feed intake} and nutrients digestibility

Concerning feed intakes (Table 2), as clearly found, rice straw intakes was linearly enhanced $(\mathrm{p}<0.05)$ by the supplementation, which raised $(\mathrm{p}<0.05)$ the total intake presented as in

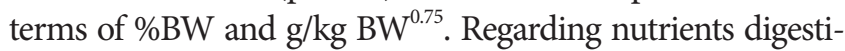
bility of DM, organic matter, $\mathrm{CP}, \mathrm{NDF}$, and $\mathrm{ADF}$ were increased $(p<0.05)$ when fed the higher level of Bamboo-Cass supplementation.

\section{Effect of Bamboo-Cass supplementation on rumen fermentation}

Rumen parameters are shown in Table 3. The rumen $\mathrm{pH}$ (6.7 to 6.8) and temperature $\left(39.2^{\circ} \mathrm{C}\right.$ to $\left.39.5^{\circ} \mathrm{C}\right)$ were in normal range. There were no influences from the dietary treatments on total VFAs ( 100.4 to $106.8 \mathrm{mmol} / \mathrm{L})$ and $\mathrm{C}_{4}(10.4 \%$ to $10.8 \%$ ), while the $\mathrm{C}_{2}$ and $\mathrm{C}_{3}$ concentration, and $\mathrm{C}_{2}: \mathrm{C}_{3}$ ratio were different among treatments $(\mathrm{p}<0.05)$. Level of BambooCass supplementation decreased $C_{2}$, while increased $C_{3}$, as well as the ratio of $\mathrm{C}_{2}: \mathrm{C}_{3}(\mathrm{p}<0.05) . \mathrm{CH}_{4}$ productions $(\mathrm{mmol} / \mathrm{L})$ were different by Bamboo-Cass supplementation $(\mathrm{p}<0.05)$. Furthermore, ruminal $\mathrm{NH}_{3}-\mathrm{N}(\mathrm{mg} / \mathrm{dL})$ concentrations were influenced $(\mathrm{p}<0.05)$, while BUN $(\mathrm{mg} / \mathrm{dL})$ was similar among treatments.

\section{Effect of Bamboo-Cass supplementation on microorganism population}

Table 4 presents the data of rumen protozoal and bacterial population as influenced by the treatments. Bacterial populations were significantly increased by increasing BambooCass supplementation, while protozoa populations were linearly reduced $(\mathrm{p}<0.05)$. Using roll-tube technique, the rumen amylolytic bacteria $(\mathrm{p}<0.05)$ and cellulolytic bacterial populations $(p<0.01)$ were enhanced, while proteolytic count remained unchanged.

\section{DISCUSSION}

\section{Chemical composition in feed ingredients}

The protein content of bamboo grass was $16 \%$ of DM, which was within the range of the previous values [9], but higher than the value in literature [7], and lower than the result of previous work [10]. However, condensed tannins and crude saponins contained in bamboo grass was $3.1 \%$ and $1.4 \%$ of DM which were higher than those in literature $[10,11]$. The differences occurred could be due to the stage of growth, drying process, harvesting method etc. 
Table 2. Effect of Bamboo-Cass on feed intake and nutrient digestibility in beef cattle

\begin{tabular}{|c|c|c|c|c|c|c|c|c|}
\hline \multirow{2}{*}{ Items } & \multicolumn{4}{|c|}{ Bamboo-Cass (g/head/d) } & \multirow{2}{*}{ SEM } & \multicolumn{3}{|c|}{$p$-value } \\
\hline & 0 & 50 & 100 & 150 & & $\mathbf{L}$ & Q & C \\
\hline \multicolumn{9}{|c|}{ Rice straw DM intake } \\
\hline $\mathrm{kg} / \mathrm{d}$ & 2.6 & 3.3 & 3.4 & 3.5 & 0.15 & 0.087 & 0.385 & 0.708 \\
\hline$\% B W$ & $1.3^{\mathrm{a}}$ & $1.6^{\mathrm{b}}$ & $1.7^{\mathrm{b}}$ & $1.7^{\mathrm{b}}$ & 0.03 & 0.025 & 0.198 & 1.000 \\
\hline \multicolumn{9}{|c|}{ Concentrate intake } \\
\hline $\mathrm{kg} / \mathrm{d}$ & 1.0 & 1.0 & 1.0 & 1.0 & 0.01 & 0.603 & 1.000 & 0.315 \\
\hline \multicolumn{9}{|c|}{ Total intake } \\
\hline $\mathrm{kg} / \mathrm{d}$ & $3.6^{\mathrm{a}}$ & $4.3^{b}$ & $4.5^{b}$ & $4.7^{\mathrm{c}}$ & 0.06 & 0.005 & 0.367 & 0.597 \\
\hline$\% B W$ & $1.8^{\mathrm{a}}$ & $2.1^{b}$ & $2.3^{c}$ & $2.3^{c}$ & 0.03 & 0.009 & 0.186 & 0.993 \\
\hline \multicolumn{9}{|c|}{ Apparent digestibility (\%) } \\
\hline DM & $62.5^{\mathrm{a}}$ & $65.6^{b}$ & $66.5^{b}$ & $66.1^{b}$ & 0.66 & 0.049 & 0.425 & 0.431 \\
\hline $\mathrm{OM}$ & $71.0^{\mathrm{a}}$ & $70.0^{\mathrm{a}}$ & $73.0^{b}$ & $72.0^{b}$ & 0.49 & 0.109 & 0.269 & 0.047 \\
\hline $\mathrm{CP}$ & $61.5^{\mathrm{a}}$ & $63.2^{b}$ & $65.0^{c}$ & $64.3^{b}$ & 0.49 & 0.833 & 0.045 & 0.881 \\
\hline NDF & $50.5^{\mathrm{a}}$ & $52.7^{b}$ & $56.6^{c}$ & $52.1^{\mathrm{a}}$ & 0.62 & 0.150 & 0.044 & 0.425 \\
\hline$A D F$ & $40.4^{\mathrm{a}}$ & $43.2^{b}$ & $46.3^{c}$ & $42.2^{b}$ & 0.45 & 0.201 & 0.034 & 0.427 \\
\hline \multicolumn{9}{|c|}{ Nutrient intake $(\mathrm{kg} / \mathrm{d})$} \\
\hline DM & 2.6 & 2.8 & 3.0 & 3.0 & 0.09 & 0.862 & 0.525 & 0.531 \\
\hline $\mathrm{OM}$ & 2.4 & 2.7 & 2.8 & 2.8 & 0.08 & 0.791 & 0.319 & 0.283 \\
\hline $\mathrm{CP}$ & 0.1 & 0.2 & 0.2 & 0.2 & 0.05 & 0.236 & 0.266 & 0.676 \\
\hline NDF & 1.5 & 1.6 & 1.9 & 1.7 & 0.08 & 0.697 & 0.328 & 0.411 \\
\hline ADF & 0.9 & 1.0 & 1.2 & 1.1 & 0.05 & 0.617 & 0.283 & 0.358 \\
\hline
\end{tabular}

SEM, standard error of mean; L, linear; Q, quadratic; C, cubic; Bamboo-Cass, Bamboo grass pellet; DM, dry matter; BW, body weight; OM, organic matter; CP, crude protein; $N D F$, neutral detergent fiber; $A D F$, acid detergent fiber.

${ }^{a-c}$ Values within the row with different superscripts are significantly different $(p<0.05)$.

Effect of Bamboo-Cass supplementation on feed intake and nutrients digestibility

The linearly increase of DM intake could be explained with the effect of Bamboo-Cass supplementation that contained additional protein and plant secondary compounds as condensed tannins and crude saponins when fed higher level of Bamboo-Cass. These findings agree with the previous studies $[25,26]$, who reported that supplementation of higher protein with suitable level of fibrous fraction would improve rumen ecology in fermentation and feed degradation, while the cellulolytic bacteria was increased. The digestibility of nutrients would be improved due to ruminal $\mathrm{pH}$, whereas microbial degradation of fiber was inhibited at $\mathrm{pH}=6.0$ or lower [27]. It enhanced ruminal $\mathrm{pH}$ (optimal 6.7 to 6.8 ) which would increase fiber degradability $[28,29]$. Furthermore, apparent nutrients digestibilities were affected by Bamboo-Cass supplementation, mainly CP, NDF, and ADF. The decrease of apparent nutrient digestibility of $\mathrm{CP}$ was correlated with low $\mathrm{NH}_{3}-\mathrm{N}$ concentration in rumen which could be explained by the effect of condensed tannins in Bamboo-Cass being bound with dietary protein as the protein-tannins complexes which would not degrade in the rumen [30]. However, NDF and ADF were also decreased by supplementation higher level. These could be explained as the effect of tannins could result lower digestibility [31]. The increase of feed intake, feed digestibility, and microbial activity was improved when degradable pro- tein was added [32].

\section{Effect of Bamboo-Cass supplementation on rumen fermentation}

The rumen $\mathrm{pH}$ is a key factor to efficient rumen fermentation by activities of microorganisms. The weak acid $\mathrm{pH}$ between 6.7-6.9 has been reported the optimal for activity of cellulolytic bacteria namely Ruminococcus albus, Ruminococcus flavefaciens, Fibrobacter succinogenes. Clearly, roughage and concentrate ratio influences ruminal $\mathrm{pH}$ and fermentation end-products with a higher supplementation level of concentrate resulting in a higher $\mathrm{pH}$ with a higher roughage intake and more $\mathrm{C}_{3}$ production [13]. The values of ruminal $\mathrm{pH}$ (6.7 to 6.8) and temperature $\left(39.3^{\circ} \mathrm{C}\right.$ to $39.5^{\circ} \mathrm{C}$ ) were similar and rather stable, which were in the normal range of rumen ecology for fermentation by rumen microbes [28,29]. Effect of Bamboo-Cass on VFA revealed that $\mathrm{C}_{2}$ and $\mathrm{C}_{4}$ were decreased while $\mathrm{C}_{3}$ increased at $150 \mathrm{~g} / \mathrm{head} / \mathrm{d}$ supplementation. This result could be explained that when $C_{2}$ decreased, $C_{3}$ could be increased while the absolute level of $\mathrm{C}_{3}$ remained unchanged [33,34]. When acetate to propionate ratio decreased, total VFAs would be decreased with supplementation with high level of dietary condensed tannins [35]. The effect of dietary condensed tannins changed propionate and acetate ratio in the in vitro study, by giving the highest propionate and low acetate to propionate ratio [36]. Furthermore, $C_{2}$ inhibitory effect could 
Table 3. Effect of Bamboo-Cass on rumen fermentation in beef cattle

\begin{tabular}{|c|c|c|c|c|c|c|c|c|}
\hline \multirow{2}{*}{ Items } & \multicolumn{4}{|c|}{ Bamboo-Cass (g/head/d) } & \multirow{2}{*}{ SEM } & \multicolumn{3}{|c|}{$\mathrm{p}$-value } \\
\hline & 0 & 50 & 100 & 150 & & L & Q & C \\
\hline \multicolumn{9}{|l|}{ Rumen pH } \\
\hline $0 \mathrm{~h}$-post feeding & 6.7 & 6.8 & 6.7 & 6.8 & 0.02 & 0.245 & 0.544 & 0.091 \\
\hline 4 & 6.7 & 6.7 & 6.6 & 6.7 & 0.02 & 0.591 & 0.291 & 0.093 \\
\hline Mean & 6.8 & 6.8 & 6.7 & 6.8 & 0.02 & 0.220 & 0.719 & 0.056 \\
\hline \multicolumn{9}{|l|}{ Temperature $\left({ }^{\circ} \mathrm{C}\right)$} \\
\hline 0 h-post feeding & 39.2 & 39.2 & 39.2 & 39.3 & 0.11 & 0.645 & 0.730 & 0.877 \\
\hline 4 & 39.5 & 39.3 & 39.3 & 39.2 & 0.07 & 0.345 & 0.242 & 0.291 \\
\hline Mean & 39.3 & 39.3 & 39.2 & 39.2 & 0.06 & 0.750 & 0.228 & 0.058 \\
\hline \multicolumn{9}{|l|}{$\mathrm{NH}_{3}-\mathrm{N}(\mathrm{mg} / \mathrm{dL})$} \\
\hline 0 h-post feeding & $14.2^{\mathrm{a}}$ & $15.6^{b}$ & $19.5^{c}$ & $13.5^{\mathrm{a}}$ & 0.44 & 0.664 & 0.006 & 0.020 \\
\hline 4 & $13.5^{b}$ & $16.7^{\mathrm{b}}$ & $13.5^{b}$ & $13.2^{\mathrm{a}}$ & 0.22 & 0.072 & 0.008 & 0.003 \\
\hline Mean & $13.9^{\mathrm{a}}$ & $16.2^{b}$ & $16.5^{b}$ & $13.4^{\mathrm{a}}$ & 0.20 & 0.519 & 0.001 & 0.442 \\
\hline \multicolumn{9}{|l|}{ BUN (mg/dL) } \\
\hline 0 h-post feeding & 13.0 & 13.0 & 13.5 & 13.5 & 0.14 & 0.172 & 1.000 & 0.468 \\
\hline 4 & 13.8 & 14.0 & 14.0 & 14.0 & 0.21 & 0.708 & 0.779 & 0.900 \\
\hline Mean & 13.4 & 13.5 & 13.8 & 13.8 & 0.17 & 0.396 & 0.859 & 0.812 \\
\hline \multicolumn{9}{|l|}{ Total VFA (mmol/L) } \\
\hline $0 \mathrm{~h}$-post feeding & 99.9 & 106.2 & 101.2 & 101.8 & 1.92 & 0.971 & 0.482 & 0.358 \\
\hline 4 & 100.9 & 107.3 & 108.2 & 105.3 & 1.55 & 0.352 & 0.189 & 0.908 \\
\hline Mean & 100.4 & 106.8 & 104.7 & 103.6 & 1.60 & 0.625 & 0.291 & 0.537 \\
\hline \multicolumn{9}{|l|}{ Acetate $\left(C_{2}\right)(\%)$} \\
\hline 0 h-post feeding & 74.8 & 70.2 & 72.1 & 70.4 & 0.62 & 0.158 & 0.336 & 0.127 \\
\hline 4 & $74.3^{b}$ & $66.1^{\mathrm{a}}$ & $67.9^{\mathrm{a}}$ & $67.5^{\mathrm{a}}$ & 0.76 & 0.034 & 0.042 & 0.124 \\
\hline Mean & $74.5^{b}$ & $68.2^{\mathrm{a}}$ & $69.6^{\mathrm{a}}$ & $68.9^{\mathrm{a}}$ & 0.47 & 0.010 & 0.022 & 0.054 \\
\hline \multicolumn{9}{|l|}{ Propionate $\left(\mathrm{C}_{3}\right)(\%)$} \\
\hline 0 h-post feeding & 14.4 & 18.2 & 16.6 & 18.5 & 0.61 & 0.249 & 0.705 & 0.184 \\
\hline 4 & $15.1^{\mathrm{a}}$ & $23.7^{\mathrm{b}}$ & $22.7^{\mathrm{b}}$ & $22.4^{b}$ & 0.72 & 0.018 & 0.019 & 0.147 \\
\hline Mean & $14.7^{\mathrm{a}}$ & $21.0^{c}$ & $19.3^{b}$ & $20.3^{b}$ & 0.56 & 0.021 & 0.052 & 0.165 \\
\hline \multicolumn{9}{|l|}{ Butyrate $\left(\mathrm{C}_{4}\right)(\%)$} \\
\hline 0 h-post feeding & 10.8 & 11.6 & 11.4 & 11.1 & 0.41 & 0.594 & 0.357 & 0.659 \\
\hline 4 & 10.6 & 10.1 & 9.4 & 10.1 & 0.13 & 0.128 & 0.069 & 0.205 \\
\hline Mean & 10.7 & 10.8 & 10.4 & 10.6 & 0.24 & 0.739 & 0.920 & 0.582 \\
\hline \multicolumn{9}{|l|}{$\mathrm{C}_{2}: \mathrm{C}_{3}$ ratio } \\
\hline 0 h-post feeding & $5.2^{c}$ & $3.9^{b}$ & $4.4^{\mathrm{b}}$ & $3.8^{\mathrm{a}}$ & 0.18 & 0.048 & 0.508 & 0.110 \\
\hline 4 & $4.9^{b}$ & $2.8^{\mathrm{a}}$ & $3.0^{\mathrm{a}}$ & $3.0^{\mathrm{a}}$ & 0.17 & 0.011 & 0.018 & 0.144 \\
\hline Mean & $4.4^{c}$ & $3.4^{\mathrm{a}}$ & $3.8^{\mathrm{b}}$ & $3.5^{\mathrm{a}}$ & 0.11 & 0.044 & 0.176 & 0.049 \\
\hline \multicolumn{9}{|c|}{ Estimated methane production (mmol/100 mol) } \\
\hline 0 h-post feeding & $34.0^{c}$ & $31.2^{\mathrm{a}}$ & $32.4^{b}$ & $31.0^{\mathrm{a}}$ & 0.33 & 0.032 & 0.169 & 0.048 \\
\hline 4 & 33.5 & 27.3 & 28.1 & 28.3 & 0.59 & 0.103 & 0.058 & 0.206 \\
\hline Mean & $33.8^{c}$ & $29.3^{\mathrm{a}}$ & $30.3^{b}$ & $28.9^{\mathrm{a}}$ & 0.32 & 0.003 & 0.051 & 0.033 \\
\hline
\end{tabular}

SEM, standard error of mean; L, linear; Q, quadratic; C, cubic; Bamboo-Cass, Bamboo grass pellet; $\mathrm{NH}_{3}-\mathrm{N}$, ammonia nitrogen; BUN, blood urea nitrogen; VFA, volatile fatty acid; $\mathrm{C}_{2}: \mathrm{C}_{3}$, acetate:propionate.

${ }^{a-c}$ Values within the row with different superscripts are significantly different $(p<0.05)$.

decrease the $\mathrm{C}_{2}$ to $\mathrm{C}_{3}$ ratio and inhibiting methanogens in the rumen [37]. This decline of estimated $\mathrm{CH}_{4}$ production $(\mathrm{p}<0.05)$, resulted by increasing of $\mathrm{C}_{2}$ to $\mathrm{C}_{3}$ ratio [37], as well as due to decreasing of protozoal population by diets containing condensed tannins [38]. In addition, the formation processes of both propionate and $\mathrm{CH}_{4}$ require $\mathrm{H}_{2}$, so the increase of propionate could be a competitive pathway with $\mathrm{CH}_{4}$ production in the rumen [22]. Supplementation of tannic acid at $6.5,13.0$, and $26.0 \mathrm{~g} / \mathrm{kg}$ of DM in beef cattle significantly decreased $\mathrm{CH}_{4}$ production from $11.1 \%, 14.7 \%$, and $33.6 \%$, respectively [36]. Tannins could play the role in inhibiting rumen methanogens' growth and activity by binding with microbial enzymes and proteins as $\mathrm{CH}_{4}$ production was decreased $[39,40]$. Moreover, $\mathrm{NH}_{3}-\mathrm{N}$ concentration was also decreased when supplemented with high level of BambooCass. This result was similar to the previous study [31] who 
Table 4. Effect of Bamboo-Cass on microbial population in beef cattle

\begin{tabular}{|c|c|c|c|c|c|c|c|c|}
\hline \multirow{2}{*}{ Items } & \multicolumn{4}{|c|}{ Bamboo-Cass (g/head/d) } & \multirow{2}{*}{ SEM } & \multicolumn{3}{|c|}{ p-value } \\
\hline & 0 & 50 & 100 & 150 & & $\mathbf{L}$ & Q & C \\
\hline \multicolumn{9}{|c|}{ Total direct count (cells/mL) } \\
\hline \multicolumn{9}{|c|}{ Protozoa $\left(\times 10^{5}\right)$} \\
\hline 0 h-post feeding & 2.8 & 2.6 & 2.6 & 2.4 & 0.11 & 0.283 & 0.779 & 0.708 \\
\hline 4 & 3.9 & 2.3 & 2.3 & 2.3 & 0.31 & 0.161 & 0.278 & 0.613 \\
\hline Mean & $3.3^{b}$ & $2.4^{\mathrm{a}}$ & $2.4^{\mathrm{a}}$ & $2.3^{\mathrm{a}}$ & 0.18 & 0.049 & 0.369 & 0.617 \\
\hline \multicolumn{9}{|l|}{ Bacteria $\left(\times 10^{11}\right)$} \\
\hline 0 h-post feeding & 28.0 & 28.3 & 28.4 & 28.6 & 0.18 & 0.495 & 0.078 & 0.072 \\
\hline 4 & $30.1^{\mathrm{a}}$ & $31.4^{\mathrm{a}}$ & $35.6^{b}$ & $37.5^{c}$ & 0.43 & 0.001 & 0.077 & 0.965 \\
\hline Mean & $29.6^{\mathrm{a}}$ & $29.9^{\mathrm{a}}$ & $32.0^{b}$ & $33.0^{c}$ & 0.29 & 0.003 & 0.066 & 0.501 \\
\hline \multicolumn{9}{|c|}{ Roll-tube technique (CFU/mL) } \\
\hline \multicolumn{9}{|c|}{ Amylolytic $\left(10^{8}\right)$} \\
\hline 0 h-post feeding & $3.1^{\mathrm{a}}$ & $3.7^{b}$ & $4.3^{c}$ & $3.1^{\mathrm{a}}$ & 0.14 & 0.738 & 0.020 & 0.200 \\
\hline 4 & $4.0^{\mathrm{a}}$ & $5.7^{b}$ & $5.8^{b}$ & $6.8^{c}$ & 0.17 & 0.002 & 0.325 & 0.146 \\
\hline Mean & $3.6^{\mathrm{a}}$ & $4.7^{b}$ & $5.1^{\mathrm{b}}$ & $4.9^{b}$ & 0.14 & 0.013 & 0.069 & 0.809 \\
\hline \multicolumn{9}{|l|}{ Proteolytic $\left(10^{8}\right)$} \\
\hline 0 h-post feeding & 2.3 & 2.4 & 1.9 & 2.2 & 0.09 & 0.417 & 0.654 & 0.137 \\
\hline 4 & $3.2^{b}$ & $2.7^{\mathrm{a}}$ & $2.9^{\mathrm{a}}$ & $3.1^{b}$ & 0.06 & 0.077 & 0.004 & 0.148 \\
\hline Mean & 2.7 & 2.6 & 2.4 & 2.7 & 0.10 & 0.675 & 0.363 & 0.638 \\
\hline \multicolumn{9}{|l|}{ Cellulolytic $\left(10^{9}\right)$} \\
\hline 0 h-post feeding & 4.8 & 4.8 & 4.9 & 5.5 & 0.10 & 0.057 & 0.204 & 0.692 \\
\hline 4 & $4.8^{\mathrm{a}}$ & $7.2^{b}$ & $8.0^{c}$ & $9.8^{d}$ & 0.14 & 0.001 & 0.322 & 0.089 \\
\hline Mean & $4.8^{\mathrm{a}}$ & $6.0^{b}$ & $6.4^{b}$ & $7.6^{c}$ & 0.12 & 0.001 & 0.831 & 0.243 \\
\hline
\end{tabular}

SEM, standard error of mean; L, linear; Q, quadratic; C, cubic; Bamboo-Cass, Bamboo grass pellet; CFU, colony-forming unit.

a-d Values within the row with different superscripts are significantly different $(p<0.05)$.

reported that condensed tannins in the diet were bound by forming protein-tannin complexation, resulting in decreasing protein degradation and $\mathrm{NH}_{3}-\mathrm{N}$ production. Additionally, it was reported that condensed tannins could bind with protein, resulting in lower $\mathrm{NH}_{3}-\mathrm{N}$ concentration [36]. This result could be due to lower $\mathrm{NH}_{3}-\mathrm{N}$ concentration from reducing protein degradability to $\mathrm{NH}_{3}-\mathrm{N}$ in the rumen, hence enhanced protein flowing to intestine [41]. However, there were no effects of saponins on feed intake or digestibility, but saponins were directly affected protozoa numbers and bacterial population by inhibiting the protozoa population and increasing bacterial population resulting a low of methane production when supplementation was at a high level [42].

\section{Effect of Bamboo-Cass supplementation on microorganism population}

Rumen protozoal population decreased, while bacterial population increased, would be due to the effect of condensed tannins and crude saponins in bamboo grass, which has the potential to suppress protozoal growth, as indicated by previous work [43]. Importantly, the increase in bacterial population and the decrease in protozoal population were found when ruminates were fed with the plants containing condensed tannins [40,44]. Moreover, supplementations of BambooCass, resulted in increasing the amylolytic bacteria, and cellulolytic bacteria while proteolytic bacterial was not affected with $150 \mathrm{~g} / \mathrm{head} / \mathrm{d}$ of Bamboo-Cass supplementation, which could be due to the decrease of protozoal population and hence, the methanogens [45].

\section{CONCLUSION}

Supplementation of Bamboo-Cass can be used as a rumen dietary enhancer as it improved rumen fermentation endproducts $\left(\mathrm{C}_{3}\right)$, reduced methane by suppressing protozoal population and increased nutrients digestibility.

\section{CONFLICT OF INTEREST}

We certify that there is no conflict of interest with any financial organization regarding the material discussed in the manuscript.

\section{ACKNOWLEDGMENTS}

The authors would like to express their most sincere thanks to Tropical Feed Resources Research and Development Center (TROFREC), Khon Kaen University, Royal Scholarship under Her Royal Highness Princess Maha Chakri Sirindhorn Education Project to the Kingdom of Cambodia, Thailand Research 
Fund (TRF) through the International Research Network (IRN) program and The Royal Golden Jubilee (TRF-IRN 57W0002) and TRF-RGJ 598001 for providing scholarship for the first author and provision of research funds and facilities.

\section{REFERENCES}

1. Hook SE, Wright ADG, McBride BW. Methanogens: Methane producers of the rumen and mitigation strategies. Archaea, 2010;2010:Article ID 945785. http://dx.doi.org/10.1155/2010/ 945785

2. Wanapat M, Kang S, Polyorach S. Development of feeding systems and strategies of supplementation to enhance rumen fermentation and ruminant production in the tropics. J Anim Sci Technol 2013;4:32. https://doi.org/10.1186/2049-1891-4-32

3. Kamra DN, Pawar M, Singh B. Effect of plant secondary metabolites on rumen methanogens and methane emissions by ruminants. In: Patra A, editor. Dietary phytochemicals and microbes. Dordrecht Netherlands: Springer; 2012. pp. 351-70.

4. Broucek J. Production of methane emissions from ruminant husbandry: a review. J Environ Prot 2014;5:1482-93. http:// dx.doi.org/10.4236/jep.2014.515141

5. Rattana S, Padungkit M, Cushnie B. Phytochemical screening, flavonoid content, and antioxidant activity of Tiliacora triandra leaf extracts. In: Proceedings of the 2nd Annual International Conference of Northeast Pharmacy Research; Maha Sarakham, Thailand: Mahasarakham University; 2010. pp. 60-3.

6. Singthong J, Ratchadaporn O, Jirawan O, Suwayd N. Bioactive compounds and encapsulation of Yanang (TILIACORA TRIANDRA) leaves. Afr J Tradit Complement Altern Med 2014;11:76-84. http://dx.doi.org/10.4314/ajtcam.v11i3.11

7. Sriket P. Chemical components and antioxidant activities of Thai local vegetables. KMITL Sci Technol J 2014;14:18-24.

8. Naibaho NM, Laohankunjit N, Kerdchoechuen O. Physicochemical properties of plant extracts from Yanang (Tiliacora triandra) leaves. J Agric Sci 2012;43:533-6.

9. Phunchago N, Wattanathorn J, Chaisiwamongkol K. Tiliacora triandra, an anti-intoxication plant, improves memory impairment, neurodegeneration, cholinergic function, and oxidative stress in hippocampus of ethanol dependence rats. Oxid Med Cell Longev 2015;2015:Article ID 918426. http://dx.doi.org/ $10.1155 / 2015 / 918426$

10. Kanpukdee S, Wanapat M. Study on ruminal degradability of local plants by using nylon bag technique. Livest Res Rural Develop 2008;20:supplement. Available from http://www.lrrd. org/lrrd20/supplement/such1.htm

11. Wanapat M, Kongmun P, Poungchompu O, et al. Effects of plants containing secondary compounds and plant oils on rumen fermentation and ecology. Trop Anim Health Prod 2012;44:399-405. https://doi.org/10.1007/s11250-011-9949-3

12. Kang S, Wanapat M, Viennasay B. Supplementation of banana flower powder pellet and plant oil sources on in vitro ruminal fermentation, digestibility, and methane production. Trop Anim Health Prod 2016;48:1673-8. https://doi.org/10.1007/ s11250-016-1142-2

13. Wanapat M, Gunun P, Anantasook N, Kang S. Changes of rumen $\mathrm{pH}$, fermentation and microbial population as influenced by different ratios of roughage (rice straw) to concentrate in dairy steers. J Agric Sci 2014;152:675-85. https://doi.org/10. 1017/S0021859613000658

14.AOAC. Official Methods of Analysis, 19th ed. Association of Official Analytical Chemists, Gaithersburg, MD, USA: AOAC International; 2012.

15. Van Soest PJ, Robertson JB, Lewis BA. Methods for dietary fiber, neutral detergent fiber, and nonstarch polysaccharides in relation to animal nutrition. J Dairy Sci 1991;74:3583-97. https://doi.org/10.3168/jds.S0022-0302(91)78551-2

16. Burns RK. Method for estimation of tannin in grain sorghum. Agron J 1971;63:511-2.

17. Kwon H, Belanger JMR, Pare JRJ, Yaylayan VA. Application of the microwave-assisted process ( $\mathrm{MAP}^{\mathrm{TM}}$ ) to the fast excretion of ginseng saponins. Food Res Int 2003;36:491-8. https://doi. org/10.1016/S0963-9969(02)00197-7

18. Samuel M, Sagathewan S, Thomus J, Mathen G. An HPLC method for estimation of volatile fatty acids of rumen fluid. Indian J Anim Sci 1997;67:805-7.

19. Galyean M. Laboratory procedure in animal nutrition research. Las Cruces, NM, USA: Department of Animal and Range Science, New Mexico State University;1989.

20. Hungate RE. A roll tube method for cultivation of strict anaerobes. In: Norris JR, Robbons DW, editors. Method in microbiology. New York, USA: Academic Press; 1969. 313 p.

21.Croker CL. Rapid determination of urea nitrogen in serum or plasma without deproteinization. Am J Med Technol 1967;33: 361-5.

22. Moss AR, Jouany JP, Newbold J. Methane production by ruminants: its contribution to global warming. Ann Zootech 2000; 49:231-53. https://doi.org/10.1051/animres:2000119

23.SAS (Statistical Analysis System). User's guide: statistic, version 9.4th edition. Cary, NC, USA: SAS Inst. Inc.; 2013.

24. Steel RGD, Torrie JH. Principles and Procedures of statistical. NY, USA: McGraw Hill Book Co; 1980.

25. Chanthakhoun V, Wanapat M, Wachirapakorn C, Wanapat M. Effect of legume (Phaseolus calcaratus) hay supplementation on rumen microorganisms, fermentation and nutrient digestibility in swamp buffalo. Livest Sci 2011;140:17-23.

26. Hung LV, Wanapat M, Cherdthong A. Effects of Leucaena leaf pellet on bacterial diversity and microbial protein synthesis in swamp buffalo fed on rice straw. Livest Sci 2013;151:18897. https://doi.org/10.1016/j.livsci.2012.11.011

27. Kang S, Wanapat M, Cherdthong A. Effect of banana flower powder supplementation as a rumen buffer on rumen fermentation efficiency and nutrient digestibility in dairy steers fed 
a high-concentrate diet. Anim Feed Sci Technol 2014;196:3241.

28. Chanjula P, Wanapat M, Wachirapakorn C, Rowlinson P. Effect of synchronizing starch sources and protein (NPN) in the rumen on feed intake, rumen microbial fermentation, nutrient utilization and performance of lactating dairy cows. AsianAustralas J Anim Sci 2004;17:1400-10. https://doi.org/10.5713/ ajas.2004.1400

29. Gunun P, Wanapat M, Gunun N, Cherdthong A, Sirilaophaisan S, Kaewwongsa W. Effects of condensed tannins in mao (Antidesma thwaitesianum Muell. Arg.) seed meal on rumen fermentation characteristics and nitrogen utilization in goats. Asian-Australas J Anim Sci 2016;29:1111-9. https:/doi.org/ 10.5713/ajas.15.0552

30.Smith AH, Zoetendal EG, Mackie RI. Bacterial mechanisms to overcome inhibitory effects of dietary tannins. Microb Ecol 2005;50:197-205. https://doi.org/10.1007/s00248-004-0180-X

31. Phesatcha B, Wanapat M, Phesatcha K, Ampapon T, Kang S. Supplementation of Flemingia Macrophylla and cassava foliage as a rumen enhancer on fermentation efficiency and estimated methane production in dairy steers. Trop Anim Health Prod 2016;48:1449-54. https://doi.org/10.1007/s11250-016-1115-5

32. Traore IA, Akouedegni GC, Babatounde S, Bosma RH. Effects of protein supplementation during the dry season on the feed intake and performances of Borgou cows in the sudanian zone of Benin. Adv Anim Biosci 2010;1:449-50. https://doi.org/10. 1017/S2040470010000725

33. Beauchemin KA, McGinn SM, Martinez TF, McAllister TA. Use of condensed tannin extract from quebracho trees to reduce methane emissions from cattle. J Anim Sci 2007;85: 1990-6. https://doi.org/10.2527/jas.2006-686

34. Makkar HPS, Becker K, Abel HJ, Szegletti C. Degradation of condensed tannins by rumen microbes exposed to quebracho tannins (QT) in rumen simulation technique (RUSITEC) and effects of QT on fermentative processes in the RUSITEC. J Sci Food Agric 1995;69:495-500. https://doi.org/10.1002/jsfa. 2740690414

35. Yang K, Wei C, Zhao GY, Xu ZW, Lin SX. Effect of dietary supplementing tannic acid in the ration of beef cattle on rumen fermentation, methane emission, microbial flora and nutrient digestibility. J Anim Physiol Anim Nutr 2017;101:302-10. https:// doi.org/10.1111/jpn.12531

36. Anantasook N, Wanapat M, Gunun P, Cherdthong A. Reducing methane production by supplementation of Terminalia chebula
RETZ. containing tannins and saponins. Anim Sci J 2016;87: 783-90. https://doi.org/10.1111/asj.12494

37. Naumann HD, Muir JP, Lambert BD, Tedeschi LO, Kothmann MM. Condensed tannins in the ruminant environment: a perspective on biological activity. J Agric Sci 2013;1:8-20.

38. Hess HD, Monsalve LM, Lascano CE, Carulla JE, Diaz TE, Kreuzer M. Supplementation of a tropical grass diet with forage legumes and Sapindus saponaria fruits: effects on in vitro ruminal nitrogen turnover and methanogenesis. Aust J Agric 2003;54:703-13.

39. Tavendale MH, Meagher LP, Pacheco D, Walker N, Attwood GT, Sivakumaran S. Methane production from in vitro rumen incubations with Lotus pedunculatus and Medicago sativa, and effects of extractable condensed tannin fractions on methanogenesis. Anim Feed Sci Technol 2005;123:403-19. https:// doi.org/10.1016/j.anifeedsci.2005.04.037

40. Tan HY, Sieo CC, Abdullah N, Liang JB, Huang XD, Ho YW. Effects of condensed tannins from Leucaena on methane production, rumen fermentation and populations of methanogens and protozoa in vitro. Anim Feed Sci Technol 2011;169:18593. https://doi.org/10.1016/j.anifeedsci.2011.07.004

41.Huang XD, Liang JB, Tan HY, Yahya R, Khamseekhiew B, Ho YW. Molecular weight and protein binding affinity of Leucaena condensed tannins and their effects on in vitro fermentation parameters. Anim Feed Sci Technol 2010;159:81-7. https:// doi.org/10.1016/j.anifeedsci.2010.05.008

42.Holtshausen L, Chaves AV, Beauchemin KA, et al. Feeding saponin-containing Yucca schidigera and Quillaja saponaria to decrease enteric methane production in dairy cows. J Dairy Sci 2009;92:2809-21. https://doi.org/10.3168/jds.2008-1843

43. Anantasook N, Wanapat M. Influence of rain tree pod meal supplementation on rice straw based diets using in vitro gas fermentation technique. Asian-Australas J Anim Sci 2012;25: 325-34. https://dx.doi.org/10.5713/ajas.2011.11131

44. Kang S, Wanapat M, Pakdee P, Pilajun R, Cherdthong A. Effects of energy level and Leucaena leucocephala leaf meal as a protein source on rumen fermentation efficiency and digestibility in swamp buffalo. Anim Feed Sci Technol 2012;174:131-9. https:// doi.org/10.1016/j.anifeedsci.2012.03.007

45. Bach A, Yoon IK, Stern MD, Jung HG, Chester-Junes H. Effects of type of carbohydrate supplementation to lush pasture on microbial fermentation in continuous culture. J Dairy Sci 1999; 82:153-60. https://doi.org/10.3168/jds.S0022-0302(99)75219-7 